\title{
Work from Home: A Primer on Managing Work- Life Balance
}

Pooja Sharma

Management Consultant, SKS Consulting \& Advisors, India.

\begin{abstract}
Work from home approach has gained significant importance recently during the COVID-19 era. Working from home provides enough time for oneself to improve upon personal cores. Work-life balance means a healthy balance between working and personal life but in the 21st century, both working and personal areas contain too much stress, anxiety, tasks, and difficulties. As an employee working remotely who is successful in making a work-life balance, one can have more productivity, fewer chances of depression, more quality time for oneself and family and happy and healthy life. In this article, we will discuss the hindrances in work-life balance and the ways to create an effective work-life balance, especially for 'work from home set-ups'.
\end{abstract}

Keywords: work from home, remote working, work-life balance, personal and working life balance

Work-life balance is balancing the work and personal lives of the employees. The efforts and hard work put into the work by employees are direct results of the level of satisfaction and fulfilment employees have in their personal lives. Work-life balance can be a daunting task in disturbing scenarios, i.e. pandemic times. With the everincreasing level of work from home adoption in the COVID-19 pandemic time, work-life balancing has become a pressing issue for management practitioners, employers, and employees alike.

\section{Why is work-life balance important?}

A positive work-life balance is the root of a successful business. If employees feel happy and motivated, they tend to provide more productivity to the business. So, the work-life balance of the employees is as fruitful for employers as it is for employees.

\section{Ways to attain work-life balance}

If you have just started working remotely, you might be feeling frustrated, tired, have less social involvement and unable to create boundaries for personal and professional tasks. 
These are simple and helpful practices to maintain a healthy work-life balance.

\section{Create boundaries}

If you have finished your working hours, don't think about your working tasks while devoting time to the family. Set your working hour timings, i.e., 9 to 5 to 10 to 6 and do not discuss work emails or business targets during the walk, meals or social gatherings. Similarly, if you have to be involved in the family tasks, be thereafter or before your working hours. Discuss this with your partner and other family members so that they do not disturb you during your working hours.

Create a routine and follow that daily. Do not change your lunchtime and tea breaks just because you are working from home. Try to follow the same routine for meals, tea, and sleep which you were following previously.

If you and your partner both are working, then work in shifts so that one is always available for the family.

\section{Find some time for personal leisure}

If you are working from home, you have enough spare time to devote to walks, yoga, meditation and for your body, because the time for getting ready for the office and travel time to reach the office can be utilized for personal wellbeing. Yoga and walking provide energy, and you feel fresh throughout the day. Similarly, you can go for a walk after dinner after sitting the whole day for work at home.

\section{Create a to-do list daily}

Maintain a diary or install an app on your mobile to write the to-do task daily. This will keep your attention and focus at work, and you will not get distracted. This will also help you to measure your level of progress daily.

\section{Separate your work area}

Create a dedicated workspace far from the bedroom so that you can physically and mentally focus on work. This is also necessary to switch from work to home mode and vice versa. Your working space should be productive and free from distractions.

\section{Take a break}

People working from home devote more time to work and forget to take a break until they complete their tasks. Don't overload yourself. Take small breaks, as you were taking earlier in the office. Go for a 15 minutes short walk, talk with your family members etc. This will also help you to refresh your mind, lower stress and improve productivity.

\section{Don't do multitasking}

Working from home doesn't mean you can complete the task of home and work at the same time. Multitasking creates distractions, reduces productivity and consumes more time. Do planning and devote time to the family and work chores according to the plans. In your breaks also, do not devote time to tasks such as laundry, cleaning, online shopping etc. 


\section{Ask for the help}

Don't hesitate to ask for help from your family members, your colleagues, or your friends. The current scenario is not the time when one person can do every task. Whenever you are stuck at any point, share with your team members or family members to find the solution collectively.

\section{Barriers in achieving work-life balance}

Even if you follow all these tips to maintain a healthy work-life balance, there may still be other obstacles that may prevent you from achieving a work-life balance if you are working remotely.

The common barriers are-

Lack of family support

Lack of team members or colleagues support

Lack of self-motivation

Poor planning

Job insecurity

Poor communication

Distractions and difficulty in switching from work mode to home mode and vice versa

Lack of coordination, direction, and supervision

To overcome these barriers, don't forget to interact, communicate and socialize. Make plans for outings and gatherings on weekends. Working from home doesn't mean you have to sit in front of a PC 24/7.

Do meditation, stretching and breathing exercises to relax your mind and body. Make schedules, work, reschedule, experiment and work. Work from home can be an opportunity to spend with our family and work as per our schedules.

\section{References}

[1]. Buchanan, D. A., \& Huczynski, A. A. (2019). Organizational behaviour. Pearson UK.

[2]. Gregory, A., \& Milner, S. E. (2009). Work-life balance: a matter of choice?. Gender, Work and Organization, 16(1), 1-13.

[3].Lockwood, N. R. (2003). Work/life balance. Challenges and Solutions, SHRM Research, USA, 2-10. 